Sadhana, Vol. 7, Part 2, July 1984, pp. 119-135. (C) Printed in India.

\title{
Vortex motion in the early stages of unsteady flow around a circular cylinder
}

\author{
LING GUO-CAN and YIN XIE-YUAN* \\ Institute of Mechanics, Chinese Academy of Sciences, Beijing. China \\ - University of Science and Technology of China, Hefei, China
}

MS received 13 April 1982; revised 12 July 1982

\begin{abstract}
Alstract. In this paper, processes in the early stages of vortex motion and the development of flow structure behind an impulsively-started circular cylinder at high Reynolds number are investigated by combining the discrete vortex model with boundary layer theory, considering the separation of incoming flow boundary layer and rear shear layer in the recirculating flow region. The development of flow structure and vortex motion, particularly the formation and development of secondary vortex and a pair of secondary vortices and their effect on the flow field are calculated. The results clearly show that the flow structure and vortices motion went through a series of complicated processes before the symmetric main vortices change into asymmetric: development of main vortices induces secondary vortices; growth of the secondary vortices causes the main vortex sheets to break off and causes the symmetric main vortices to become "free" vortices, while a pair of secondary vortices is formed; then the vortex sheets, after breaking off, gradually extend downstream and the structure of a pair of secondary vortices becomes relaxed. These features of vortex motion look very much like the observed features in some available flow field visualizations. The action of the secondary vortices causes the main vortex sheets to break off and converts the main vortices into free vortices. This should be the immediate cause leading to the instability of the motion of the symmetric main vortices. The flow field structure such as the separation position of boundary layer and rear shear layer, the unsteady pressure distributions and the drag coefficient are calculated. Comparison with other results or experiments is also made.
\end{abstract}

Keywords. vortex motion; unsteady flow; circular cylinder; flow structure; boundary layer separation; rear shear layer separation; viscous vortex core

\section{Introduction}

The problem of the unsteady motion of a circular cylinder impulsively started from rest and subsequently moving with a constant velocity perpendicular to its axis is a classical problem in fluid dynamics. With the passage of time, it exhibits a series of complicated flow phenomena, especially at high Reynolds numbers. Over the years, much progress has been made in some aspects of this problem with various degrees of success, such as unsteady boundary layer separation in the early stages of motion, numerical solutions of the N-S equations for flow field at low and moderate Reynolds numbers and the modeling of vortex street by use of discrete vortices at high Reynolds numbers. Some general aspects of the flow around a circular cylinder at different ranges of Reynolds numbers have also been known. But for the early stages of unsteady motion, i.e. from the beginning of boundary layer separation to the time of establishment of Karmán vortex street, details of the process of the formation of vortices and the evolution of flow structure with time are not yet clearly understood. Especially in the situation of 
high Reynolds number, a satisfactory explanation and description about the following problems and phenomena have not yet been given theoretically: (i) The formation of secondary vortex and a pair of secondary vortices, the evolution of the secondary vortices with time and the effect of the secondary vortices on the symmetric main vortices (ii) The process of development and shedding of symmetric main vortices. Under what condition will the motion of the symmetric main vortices become unstable? or what is the immediate cause that leads to the instability of the motion of symmetric vortices? (iii) The variations of flow field with time. Although at low and moderate Reynolds number, the details of whole flow field structure can be obtained using numerical solution of the $\mathrm{N}-\mathrm{S}$ equations, at high Reynolds number there are considerable difficulties to do so, only a few numerical results are available. Among those numerical analyses, some studies (Thoman \& Szewczyk 1969; Son \& Hanratty 1969; Collins \& Dennis 1973; Patel 1976; Ta Phouc Loc 1980) are related with secondary vortex. Thoman \& Szewczyk (1969) have given the forming and changing of main vortices and secondary vortices at $\operatorname{Re} \sim 4 \times 10^{4}$. At moderate Reynolds numbers Son \& Hanratty (1969) and Collins \& Dennis (1973) have calculated a single secondary vortex structure, which is a slight "swelling" of local streamlines. The vortex pattern is not obvious. These studies were concerned with the near wake evolution and other characters of flow field, and did not concentrate on the investigation of secondary vortices. A recent numerical analysis of the $\mathrm{N}-\mathrm{S}$ equations about secondary vortices has been given by Ta Phouc $\operatorname{Loc}(1980)(\operatorname{Re}=300,590,1000)$. It seems to be not easy to give a clear physical picture of the whole process with numerical analysis of the $\mathrm{N}-\mathrm{S}$ equations at high Reynolds number.

The discrete vortex model has been used to investigate the vortex motion by many authors with varying degrees of success (Rosenhead 1931; Gerrard 1967; Sarpkaya \& Shoaff 1979; Chorin 1973; Moore 1974; Fink \& Soh 1974; Clements 1977; Deffenbaugh \& Marshall 1976; Kunio 1978; Stansby 1981). It also has been reviewed by others (Saffman \& Baker 1979; Clements \& Maull 1975; Bearman \& Graham 1980). Recently, using the method of combining discrete vortex model with boundary layer theory to study unsteady separated flow around a circular cylinder at high Reynolds numbers, in some aspects of the problem, such as predictions of the drag and lift coefficients, boundary layer separation and modelling of the vortex street in the wake etc., successes in varying degrees have been gained by some authors, (e.g. Sarpkaya \& Shoaff 1979; Deffenbaugh \& Marshall 1976; Kunio 1978; Stansby 1981). But these results are mainly related to the flow features at a long time. Most investigations do not deal with the secondary vortices and the separation of rear shear layer as well as the processes of vortices motion in the early stages of motion, although Deffenbaugh \& Marshall (1976) calculated the rear shear layer separation by empirical formula.

Using careful experimental techniques, flow visualizations of unsteady wake development behind the circular cylinder in different Reynolds number ranges have been made by some authors (Hiroyuki \& Sadatoshi 1969; Taneda 1972; Nagata et al 1975; Coutanceau \& Bouard 1980). At $500<\operatorname{Re}<10^{4}$, the formation of secondary vortices and the development of main vortices have been observed carefully. It has been shown that there is a series of complicated processes during the formation of Karman vortex street. All these phenomena give us a new insight into the mechanism of the formation of Karman vortex street.

In the present work an attempt has been made to describe and formulate the vortices motion and flow structure development with time before the symmetric main vortices 
change into asymmetric, to analyse the early processes of the formation of Karman vortex street, using some methods simpler than the numerical analysis of the $\mathrm{N}-\mathrm{S}$ equations but capable of showing the basic features of vortex motion during the early stages of unsteady motion. In the present work, based on the studies of Ling \& Yin $(1981,1982)$ a method is used that combines the discrete vortex model with boundary layer theory and considers the separation of boundary layer together with the separation of the rear shear layer. In order to avoid singularity of velocity induced by potential vortex theory, viscous vortex core has been used. In the present work the development of vortices motion, the development of boundary layer separation, and the drag coefficient, etc are described and calculated. Especially attention is paid to the formation and growth of secondary vortices in the recirculating region, to the effect of secondary vortices on the symmetric main vortices and on the flow field, to the role they play in leading the motion of symmetric main vortices to instability. In order to understand the flow evolution, the pressure and velocity distributions on surface are also given. In the present work laminar flow and high Reynolds number are considered.

\section{Theoretical analysis}

\subsection{Model and method}

In the present work a method is used that combines the discrete vortex model with boundary layer theory. It is well known, that at high Reynolds number, the thicknesses of the boundary layer and the shear layer produced by separation of boundary layer are thin, the shear layer may be considered a vortex sheet. From flow field analysis (Ling \& Yin 1981) and visualizations, we know that in the early stages of unsteady motion, a recirculating shear layer is gradually formed close to the surface behind the cylinder. The maximum value of recirculating potential flow velocity on the surface may be of the same order of magnitude as the incoming flow. It follows that the rear shear layer is also rather thin. Under certain condition, it will separate and form a thin vortex sheet. The effect of viscosity is primarily limited to these thin layers. According to the ideas of discrete vortex model the continuous distributions of vorticity in the vortex sheets may be represented by a series of discrete vortices having certain strengths. The vorticity both in the boundary layer and in the rear shear layer are transported continuously into the near wake through their own separation points. This continuous transport process may be represented approximately by the shedding of discrete vortices and their subsequent motion. We assume that the vortex point moves with local spatial mean velocity which is also the local velocity of the flow field in the absence of the vortex. The whole potential flow field is formed by superposition of all discrete vortices, their images about the circular cylinder, uniform flow and doublet. Thus, the motion of each discrete vortex and the velocity as well as the pressure distributions on the surface can be obtained at any instant. The velocity and pressure distributions give the conditions at outer edges of the boundary layer and rear shear layer for calculating their separation in the next instant, then we can calculate the new separation points and determine the new discrete vortices. After that we can obtain a new potential flow field and new distributions of velocity and pressure. The procedure described above is performed repeatedly. This is the model that we used to investigate the early stages of unsteady motion of circular cylinder at high Reynolds number; this method includes the interactions between boundary layer, external flow and near wake. 


\subsection{Outer flow}

The flow outside the regions of boundary layer, rear shear layer and the vortex core is assumed to be a potential flow. The velocity potential function is subject to Laplace equation. The complex potential function of the entire potential flow field, which is made up of the uniform flow over a circular cylinder and a series of discrete vortices outside the circular cylinder, may be given by means of the circle theorem. When a discrete vortex is shedding from the cylinder, an equivalent circulation with opposite sign as the shed one is added onto the surface of the cylinder so that the total vorticity in the potential flow field remains conserved. Let $W=\tilde{W} / R V_{\infty}$ represent the nondimensional complex potential function, $\phi=\tilde{\phi} / R V_{\infty}$-potential function of velocity, $\psi=\tilde{\psi} / R V_{\infty}$-stream function, $\Gamma=\tilde{\Gamma} / 2 \pi R V_{\infty}$-circulation, $V=\tilde{V} / V_{\infty}$-velocity, $t=V_{\infty} \grave{t} / R$-time, $C_{P}=\left(P-P_{\infty}\right) / \frac{1}{2} \rho V_{\infty}^{2}$-pressure coefficient, $C_{D}=D / \frac{1}{2} \rho V_{\infty}^{2} R-$ form drag coefficient, $C_{L}=L / \frac{1}{2} \rho V_{\infty}^{2} R$-lift coefficient. $R$ represents the radius of the cylinder. $D$ and $L$ represent, respectively, the drag and lift force acting on the cylinder. Subscript $\infty$ refers to the incoming flow. Then we have

$$
\begin{aligned}
W(z) & =z+\frac{1}{z}+\sum_{n=1}^{N} i \Gamma_{n} \ln \frac{z-\frac{1}{\bar{z}_{n}}}{z-z_{n}}, \\
\phi(r, \theta) & =\frac{r^{2}+1}{r} \cos \theta-\sum_{n=1}^{N} \Gamma_{n} \theta^{* *}+\sum_{n=1}^{N} \Gamma_{n} \theta^{*}, \\
\psi(r, \theta) & =\frac{r^{2}-1}{r} \sin \theta+\sum_{n=1}^{N} \frac{\Gamma_{n}}{2} \ln \frac{R_{1}}{r_{n}^{2} R_{2}},
\end{aligned}
$$

here $r, \theta$ are polar coordinates, $\theta$ is measured from the rear stagnation point.

The velocity components of the flow field in polar coordinates are obtained:

$$
\begin{aligned}
V_{r}(r, \theta)= & \frac{r^{2}-1}{r^{2}} \cos \theta+\sum_{n=1}^{N} \Gamma_{n} \frac{r_{n} \sin \left(\theta-\theta_{n}\right)}{R_{1}}-\sum_{n=1}^{N} \Gamma_{n} \frac{r_{n} \sin \left(\theta-\theta_{n}\right)}{R_{2}} \\
V_{\theta}(r, \theta)= & -\frac{r^{2}+1}{r^{2}} \sin \theta-\sum_{n=1}^{N} \Gamma_{n} \frac{r r_{n}^{2}-r_{n} \cos \left(\theta-\theta_{n}\right)}{R_{1}} \\
& +\sum_{n=1}^{N} \Gamma_{n} \frac{r-r_{n} \cos \left(\theta-\theta_{n}\right)}{R_{2}} .
\end{aligned}
$$

In formulae (1) and (2),

$$
\left.\begin{array}{rl}
\theta^{* *} & =\operatorname{tg}^{-1} \frac{r r_{n} \sin \theta-\sin \theta_{n}}{r r_{n} \cos \theta-\cos \theta_{n}}, \\
\theta^{*} & =\operatorname{tg}^{-1} \frac{r \sin \theta-r_{n} \sin \theta_{n}}{r \cos \theta-r_{n} \cos \theta_{n}}, \\
R_{1} & =1+r^{2} r_{n}^{2}-2 r r_{n} \cos \left(\theta-\theta_{n}\right), \\
R_{2} & =r^{2}+r_{n}^{2}-2 r r_{n} \cos \left(\theta-\theta_{n}\right) .
\end{array}\right\}
$$


The equations of motion of the $k$-th discrete vortex are

$$
\begin{aligned}
\frac{\mathrm{d} r_{k}}{\mathrm{~d} t}= & \frac{r_{k}^{2}-1}{r_{k}^{2}} \cos \theta_{k}+\sum_{n=1}^{N} \Gamma_{n} \frac{r_{n} \sin \left(\theta_{k}-\theta_{n}\right)}{S_{1}}-\sum_{n \neq k}^{N} \Gamma_{n} \frac{r_{n} \sin \left(\theta_{k}-\theta_{n}\right)}{S_{2}} \\
\frac{\mathrm{d} \theta_{k}}{\mathrm{~d} t}= & -\frac{r_{k}^{2}+1}{r_{k}^{3}} \sin \theta_{k}-\sum_{n=1}^{N} \Gamma_{n} \frac{r_{k} r_{n}^{2}-r_{n} \cos \left(\theta_{k}-\theta_{n}\right)}{r_{k} S_{1}} \\
& +\sum_{n \neq k}^{N} \Gamma_{n} \frac{r_{k}-r_{n} \cos \left(\theta_{k}-\theta_{n}\right)}{r_{k} S_{2}} \\
S_{1}= & R_{1}\left(r_{k}, \theta_{k}, r_{n}, \theta_{n}\right), \quad S_{2}=R_{2}\left(r_{k}, \theta_{k}, r_{n}, \theta_{n}\right), \\
(n= & 1,2, \ldots, N ; \quad k=1,2, \ldots, N) .
\end{aligned}
$$

In equations (1) to (3) $N$ is the total number of all discrete vortices, i.e. both the vortices from the boundary layer separation and the vortices from the rear shear layer separation. $\Gamma_{n}$ is the strength of $n$th vortex, its value will be given in a later paragraph.

The pressure distribution on the cylinder surface and the form drag and lift coefficients are given:

$$
\begin{aligned}
C_{P}(\theta, t) & =1-V_{B}^{2}+2 \sum_{n=1}^{N} \Gamma_{n} \frac{\partial}{\partial t}\left(\theta_{r=1}^{* *}-\theta_{r=1}^{*}\right), \\
C_{D} & =-4 \pi \sum_{k=1}^{N} \Gamma_{k}\left\{\left(1+\frac{1}{r_{k}^{2}}\right) \frac{\mathrm{d} r_{k}}{\mathrm{~d} t} \sin \theta_{k}+\left(r_{k}-\frac{1}{r_{k}}\right) \frac{\mathrm{d} \theta_{k}}{\mathrm{~d} t} \cos \theta_{k}\right\}, \\
C_{L} & =4 \pi \sum_{k=1}^{N} \Gamma_{k}\left\{\left(1+\frac{1}{r_{k}^{2}}\right) \frac{\mathrm{d} r_{k}}{\mathrm{~d} t} \cos \theta_{k}-\left(r_{k}-\frac{1}{r_{k}}\right) \frac{\mathrm{d} \theta_{k}}{\mathrm{~d} t} \sin \theta_{k}\right\}
\end{aligned}
$$

\subsection{Boundary layer separation and rear shear layer separation}

The flow near the cylinder surface, called inner flow, should be governed by boundary layer equations of two-dimensional incompressible flow having appropriate boundary conditions and initial conditions. The boundary conditions are given by potential flow field as mentioned in the paragraph above. So the outer flow and the inner flow would be solved simultaneously. In discrete vortex model, only the positions of separation point of boundary layer or rear shear layer are of interest, while the details of inner flow are not necessary. Therefore, the emphasis is placed on the determination of development of separation points. Having observed the development of boundary layer, it is found that although the external flow changes continuously with time, the change of boundary layer separation point quickly approaches a quasi-steady state after a very short period $t \doteq 1$, after initial separation (Sarpkaya \& Shoaff 1979; Deffenbaugh and Marshall 1976). From then on the boundary layer separation point may be calculated assuming quasi-steady flow.

In the very early stages of unsteady motion, $t<1$, it is more reasonable to determine the separation points by Moore-Rott-Sears (MRS) criterion (Sears \& Telionis 1975) and to place the first and the subsequent nascent discrete vortices on the separation points obtained by this method because the separation points predicted by this criterion represent the beginning of a wake or separated "bubble". The method of calculating separation point by MRS criterion is a numerical analysis, by which the singularity of 
unsteady boundary layer equations is determined, and it is not repeated in the present work. The variation of separation points has been given by Telionis \& Tsahalis (1973). In the region of $t \leqslant 1$, this variation may be represented approximately by an empirical formula given in part (4) of the text by the present authors. According to Telionis \& Tsahalis (1973) the first separation positions are near $\theta= \pm 40^{\circ}$ at $t=0.65$. When $t>1$ there is no substantial difference in the prediction of boundary layer separation points either with MRS criterion or with Prandtl criterion.

The effects of discrete vortices on the flow, which are shed before the boundary layer becomes quasi-steady state, have been analyzed by Ling \& Yin (1981). It is shown that in two situations, i.e. one calculation starting from $t=0.65$ and the other from $t=1$, the calculated positions of vortex motion, the velocity or pressure distributions on the cylinder near the rear stagnation point are different. But these differences are local, for the most part of the cylinder surface the difference is slight. In addition, the transition of separation point, from $t<1$ (calculated with MRS criterion) to $t>1$ (calculated with Prandtl criterion) should be smooth at the junction, otherwise the spiral shape of the vortex will be broken after certain instant. This would be disadvantageous for analyzing the vortices motion and the development of flow structure in the early stages.

Based on previous studies, for simplicity, the boundary layer separation pointcoupled calculation in the present work is started from the situation of boundary layer approaching quasi-steady state, i.e. from $t=1$. Considering the flow quantities dependent on time, the position with maximum velocity and the position with minimum pressure on the surface may not coincide at all instants. The Stratford method (White 1974) is extended and used to calculate the separation point. The following relations would be satisfied at separation point:

$$
\begin{aligned}
& {\left[C_{P}^{\prime}\left(x^{\prime} \frac{\mathrm{d} C_{P}^{\prime}}{\mathrm{d} x^{\prime}}\right)^{2}\right]_{s}=0.0104} \\
& x^{\prime}=\theta-\theta_{B L} \\
& \theta_{B L}=\theta_{P \min }-\int_{0}^{\theta_{P \min }}\left(V_{B} / V_{B}^{*}\right)^{5} \mathrm{~d} \theta \\
& C_{P}^{\prime}=\left(P-P_{\min }\right) / \frac{1}{2} \rho\left(V_{B}^{*}\right)^{2}
\end{aligned}
$$

where $P_{\min }$ is the minimum pressure on the cylinder, $V_{B}^{*}$ the potential velocity at outer edge of boundary layer at the position $P=P_{\min }$.

The unsteady flow in the early stages has been analysed by Ling and Yin (1981) in which the secondary vortex is not considered. The same authors have shown that with the evolution of symmetric main vortices and under their effect, a recirculating flow region is gradually formed behind the cylinder, the region gradually extends and reaches the rear stagnation point. At that time, behind the cylinder and near the surface, a rear shear layer including rear stagnation point has formed. With the evolution of time, the maximum of recirculating potential velocity on the cylinder may reach the same order of magnitude as that of the incoming flow. The recirculating flow along the cylinder surface undergoes partly the action of adverse pressure gradient. It is postulated that in this early stage, the nature of the rear shear layer is similar to that of a laminar boundary layer. It will separate under the continuous action (no instantaneous action) of adverse pressure gradient. Under the circumstance in which the position of minimum pressure on the surface in recirculating flow region no longer moves forward, 
the separation of rear shear layer is taken into account. At this time the quasi-steady state is also postulated. Using the method expressed in equation (5), the rear shear layer separation point can be calculated.

The strength of a discrete vortex is assumed to be equal to the vorticity flux across the section of boundary layer at the separation point in a time increment. If $\partial \Gamma / \partial t$ represents the vorticity flux, $v_{\theta}$, the circumferential velocity in boundary layer and $V_{B S}$, the potential velocity at separation point, then we have

$$
\begin{aligned}
& \partial \Gamma / \partial t=\frac{1}{2 \pi}\left[\int_{0}^{\delta} \frac{\partial v_{\theta}}{\partial r} v_{\theta} \mathrm{d} r\right]_{s}, \\
& \Gamma_{n}^{f}=\frac{+1}{4 \pi} V_{B S}^{2} \Delta t,
\end{aligned}
$$

where $\Delta t$ is the time increment, $\Gamma_{n}^{f}$ is the strength of $n$th discrete vortex shedding from boundary layer.

The strength of secondary discrete vortex, $\Gamma_{n}$, shedding from the rear shear layer is

$$
\Gamma_{n}^{r}=\mp \frac{1}{4 \pi} V_{B S_{r}}^{2} \Delta t
$$

where $V_{B S_{\gamma}}$ is the potential velocity at the position of rear shear layer separation point. From formulae (6), it is shown that the strength of discrete vortex only directly depends on the potential velocity at the outer edge of the boundary layer at separation point, it does not depend on the detaits of inner flow directly.

In order to consider reasonably the reduction of circulation of the vortices in the practical wake flow, we can multiply the strength of discrete vortices by a factor $\sigma$, which is less or equal to one. The value of $\sigma$ can only be determined by experiments or numerical experiments at present.

\subsection{Viscous vortex core}

In the potential flow theory, near the centre of vortex the induced velocity is not reasonable in reality, at the centre of vortex it approaches infinity. In order to remove this singularity we introduce a viscous core to describe the actual flow near the vortex. From the exact solution for the decay of an axially symmetric vortex (Lamb 1945) one can evaluate the magnitude of core (at the edge of core the velocity has maximum) and calculate the induced velocity in the inner region. Let $V_{j, k}$ represent the induced velocity by $j$ th small vortex at the position of $k$ th point, $l_{j, k}$ represent the distance between $j$ th vortex and $k$ th point, $r_{c j}, \Delta t_{j}$ represent the radius of $j$ th vortex core and its "age" respectively, $r_{c j o}$ represent the initial radius of $j$ th vortex core. Then, we have

$$
\begin{aligned}
& V_{j, k}=\frac{\Gamma_{j}}{l_{j k}}\left[1-\exp \left(-1 \cdot 256 \frac{l_{j k}^{2}}{r_{c j}^{2}}\right)\right], \\
& r_{c j}=r_{c j o}+3 \cdot 17\left(\Delta t_{j} / \operatorname{Re}_{D}\right)^{1 / 2} .
\end{aligned}
$$

The initial radius of vortex core is given by referring to the thickness of boundary layer where the discrete vortex is shed. $\mathbf{R e}_{D}$ is a Reynolds number, in which the characteristic velocity is incoming flow velocity $V_{\infty}$. 


\subsection{Calculation scheme}

Formulae (1) to (7) are simultaneous equations for determining the motion and strength of $N$ discrete vortices as well as the flow field. The simultaneous ordinary differential equations may be solved by numerical integration of Runge-kutta having appropriate initial conditions. The positions of separation are calculated by the numerical integration method of Simpson as well as the method of linear interpolation. Shedding of vorticity at the shed point will make local flow field distort and promote an earlier separation of the boundary layer (Sarpkaya \& Shoaff 1979). In order to alleviate these influences the induced action by any new discrete vortex will be considered after a time step immediately after it was shed. The initial radial position of nascent discrete vortex, which has to be a small distance apart from the cylinder surface, may be determined by different methods, such as no slip condition on the separation point at shedding moment (Sarpkaya \& Shoaff 1979; Deffenbaugh \& Marshall 1976) or from the requirement of calculation stability (Kunio 1978; Yang \& Bar-Lev 1976) or other method (Stansby 1981). For simplicity, in the present work, the initial radial position is taken as $r_{0}=1.05$ referring to Yang \& Bar-Lev (1976).

In calculations of work ( $\mathrm{Ling} \&$ Yin 1981, 1982), different time steps, $\Delta t=0 \cdot 1,0 \cdot 125$, $0 \cdot 2$, have been used. The calculation results with these time steps have shown that the form drag coefficients, positions of boundary layer separation and positions of spiral vortices did not differ significantly, the pressure and velocity distributions on surface also did not significantly differ, except for several positions of singularity produced by several discrete vortices which are extremely adjacent to the surface. In general, if the time step is too large, the "discrete" method will not effectively describe the continuous distribution or continuous transport of vorticity, while if it is too small, an apparent problem is the computer storage and time will increase rapidly. In the present work, equal time step $\Delta t=0.125$ is used.

For the calculation beginning at the boundary layer reaching quasi-steady state, i.e. at $t=1$, the initial circumferential positions of nascent vortices are placed at $\theta= \pm 75^{\circ}$, they are positions of separation points solved from equations (5) by the present work. From the start of the computation after each time step the discrete vortices increase in number by two, the simultaneous equations increase in number by six. When the position of minimum pressure on the surface in recirculating flow region no longer moves forward, the rear shear layer separation begins to calculate. From then on, after each time step discrete vortices increase in number by four, simultaneous equations increase in number by twelve. At $t=5$, the number of total discrete vortices will reach 98 with 294 simultaneous equations governing their motion.

\section{Results}

In the present work the process of vortices motion and development of flow structure behind a cylinder in the early stages have been investigated. During this period the motion of main vortices is symmetrical. The development of vortices motion, the change of position of separation, the pressure and velocity distributions on the surface as well as the drag coefficient are given. In this paper only part of the results are given. The case is that the first nascent vortices were generated at $t=1, \Delta t=0.125, \sigma=0.39$ (only for rear vortices) and $\operatorname{Re}_{D}=10^{4}$ in formula (7). The results for other cases and 
different parameters are given in work (Ling \& Yin 1981, 1982). In the text some comparisons will be noted.

Figures 1-12 describes the development of vortices, especially the generation and growth of secondary vortices and their effects, in detail (in each figure, a right quarter diagram is drawn). Starting from $t=1$, with the evolution of time the discrete vortices shed into near wake and form two symmetric vortex sheets. At $t=2$ two vortex sheets have rolled up to form a pair of nearly spiral symmetric vortices. With increasing time, more and more discrete vortices join the sheets and the rolls of vortices grow bigger as shown in figures 1 to 4 . Under the influence of the main symmetric vortices, at $t=3.25$ the rear shear layer begins to separate and the secondary discrete vortices i.e. rear discrete vortices, start to shed. From figures 5 to 8 it can be seen that the secondary discrete vortices increase gradually in number and form the secondary vortex sheet in a finite region. The strength of its vorticity also gradually increases. The secondary vortices obviously affect the flow field, the motion of discrete vortices generated from the boundary layer separation, the vorticity transport and the vorticity accumulation in the symmetric main vortices. At some instant, the symmetric main vortex sheet splits into two parts, as shown in figures 7 and 8 . The disconnected vortex sheet will roll up again at the end and become the third vortex sheet. A pair of secondary vortices is made up of the third vortex and the secondary one in a finite region, as shown in figure 8. On the other hand, the main vortex becomes a free vortex having no more supplement in vorticity. From then on, with time increasing the vortex sheet attached to the boundary layer gradually expands downstream again as shown in figures 9 to 12 . The main vortex sheets, having been broken into two parts, show a tendency of re-connecting, while the structure of the secondary vortex roll gradually becomes relaxed. As shown above, in the period considered in the present work the variation of flow structure and vortices motion undergo a series of complicated processes. It is worthy of remark that the transition of two symmetric vortices from a situation with vorticity addition into the situation of a pair of free vortices having no more supplement in vorticity suggests that an unstable condition of the motion of the symmetric main vortices has just appeared. At this time under the action of small disturbances the fluctuation of the vortices may be easily produced and the symmetric main vortices tend to asymmetric motion. The action of secondary vortices causing the symmetric main vortices to become a pair of free vortices is the immediate cause that leads to the instability of the motion of the pair of symmetric vortices. Figures 13 to 18 are some flow visualizations of Nagata et al (1975), in which $\operatorname{Re}_{D}=1220$. These figures also show the development and the effect of secondary vortices, the process of breaking of the main vortex sheets and the formation of a pair of secondary vortices. In the other plates $t>6.2$ (see Nagata et al. 1975), the motion of the pair of main vortices has actually become asymmetric. Comparing the above results, it is clear that the features of vortices motion given in the present work look very much like the flow visualizations. The processes of vortices motion and variations of flow structure now given are in good agreement with other experimental observations. (Hiroyuki \& Sadatoshi 1969; Coutanceau \& Bouard 1980) ( $\alpha$ phenomena, $\operatorname{Re} 800 \sim 5000$ ). Similar processes where secondary vortex grows and where the main vortex is split into two parts, were also obtained in the numerical solution of the $\mathrm{N}-\mathrm{S}$ equations by Thoman \& Szewczyk (1969) at $\operatorname{Re} 4 \times 10^{4}$. But in another experiment (Coutanceau \& Bouard 1980), in much higher Reynolds number (say $\operatorname{Re~} \sim 9500$ ), the flow in the near wake becomes unstable, and these processes obtained in the present work have not been observed. Figure 19 shows results of another computation by Ling 

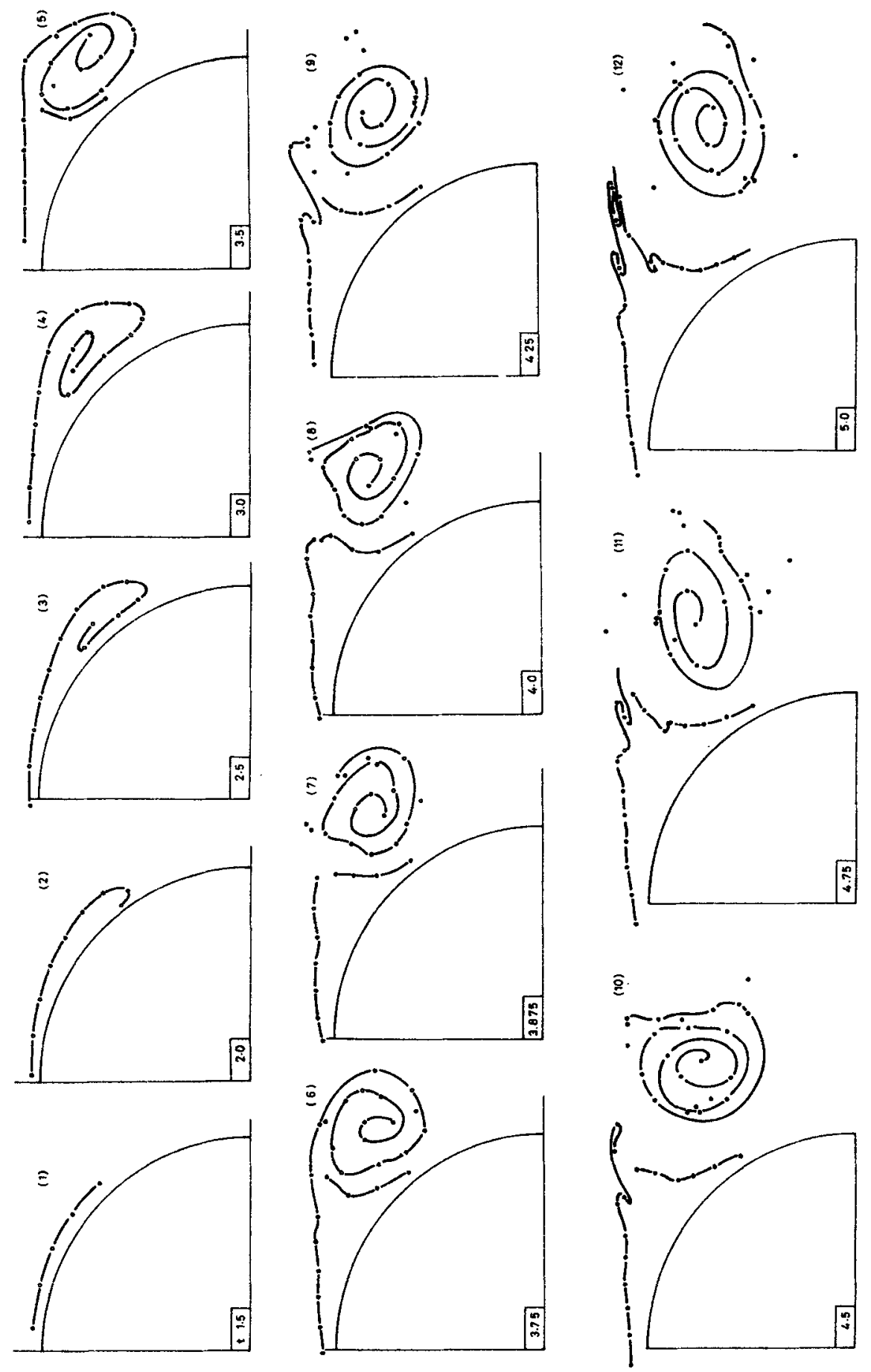

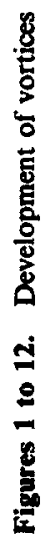
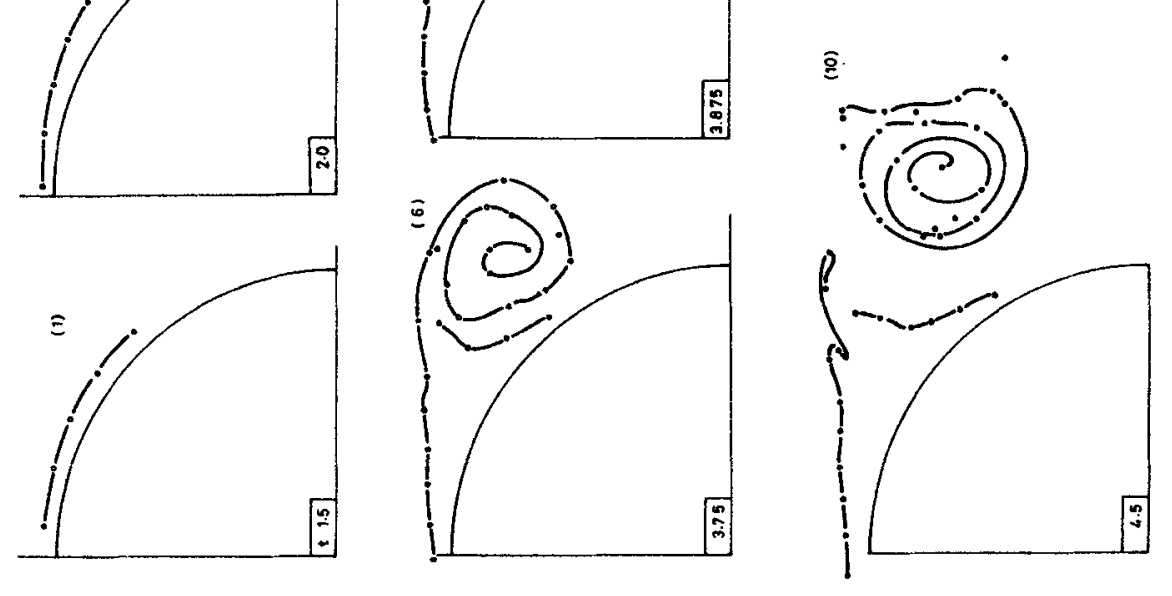

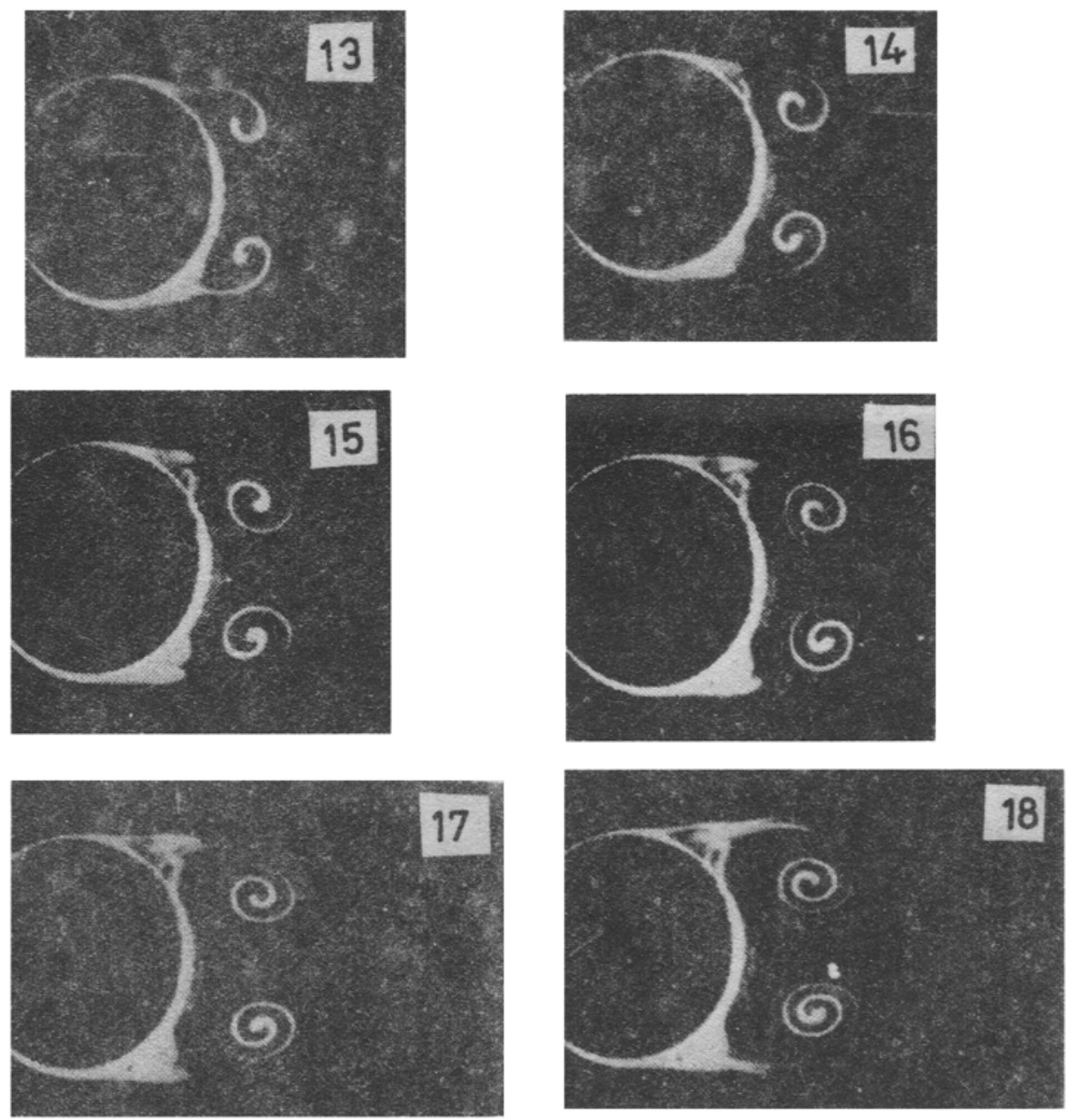

Figures 13 to 18. Vortex development visualization behind a circular cylinder. 13. $t: 3$; 14. $t: 3 \cdot 4 ; 15 . t: 3 \cdot 8 ; 16$. $t: 4 \cdot 6 ; 17 . t: 5 \cdot 2 ; 18 . t: 6 \cdot 2$ (after Nagata et at 1975)

\& Yin (1982). The variation of boundary layer separation points is given by an empirical formula i.e. $\theta_{s}=98-58 \exp (-1.1968(t-0.65))$, referring to the results of work (Thoman \& Szewczyk 1969; Deffenbaugh \& Marshall 1976; Telionis \& Tsahalis 1973). The first separation takes place at $t=0.65$ and $\theta_{s}= \pm 40^{\circ}$. The rear shear layer separation is calculated by formula (5). The parameters $\Delta t=0.125, \sigma=1$ and no viscous vortex core is considered. Comparing figures 1-12 with figure 19 , these results described the same processes of vortices motion and development of flow structure although the positions of vortices shown in the figures do not coincide well with each other at the corresponding instant. It seems to show that neglecting the effect of discrete vortices shed before $t=1$ does not change the nature of vortices development in the early stages.

The variation of boundary layer separation points is plotted in figure 20 . It is a coupled computation result containing interactions between boundary layer, near 

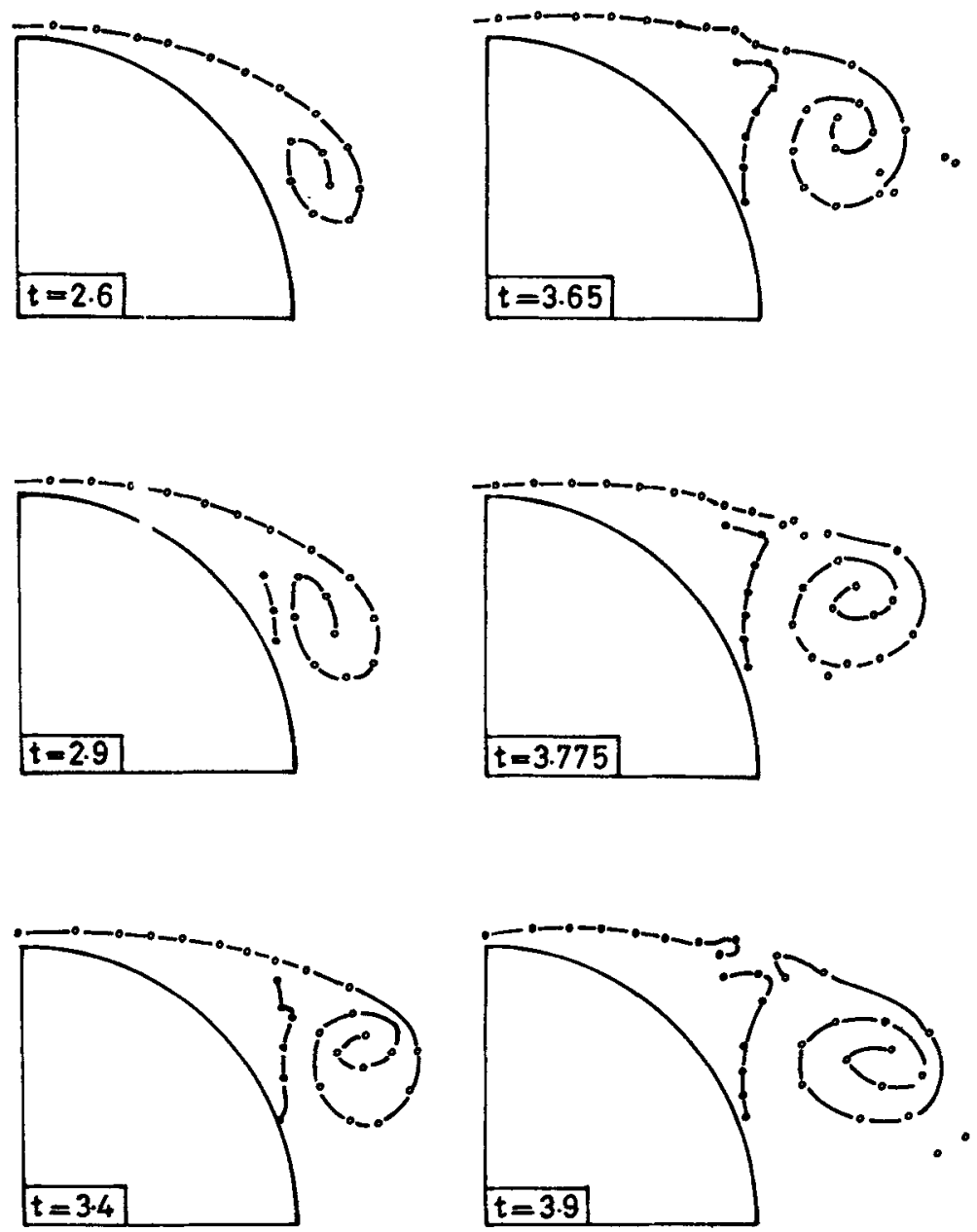

Figure 19. Development of vortices

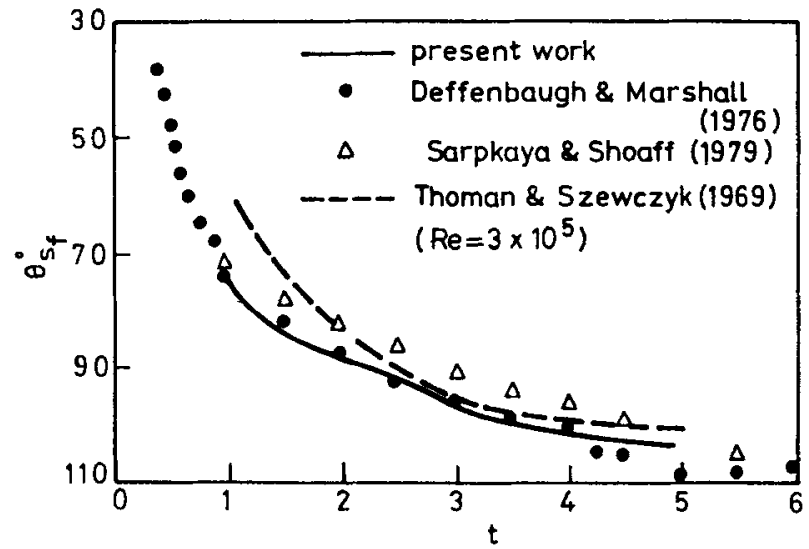

Figure 20. Transient boundary layer separation point. 
wake and external flow. With evolution of time, the separation point moves forward quite fast at first, then approaches its steady position gradually. The result is in good agreement with the result of Deffenbaugh \& Marshall (1976) when $t<4$. After a rather long time, the separation angle from front stagnation point reaches $77^{\circ}$ which is close to some results (Sarpkaya \& Shoaff 1979; White 1974). The calculation result is rather less than the numerical results of the $\mathrm{N}-\mathrm{S}$ equations. Variation of rear shear layer separation point is plotted in figure 21. After first separation, the separation angle gradually decreases with time. This tendency is reasonable. The positions of rear separation points are rather high as compared with those reported by Ling \& Yin (1982), unfortunately neither experimental results nor exact solutions are available for comparison.

The change of pressure distributions on the surface with time is plotted in figure 22 . Due to separation of boundary layer and formation of vortices, the pressure distributions are different from that obtained in potential unseparated flow, especially on the rearward surface of the cylinder. The pressure at forward stagnation point also changes slightly. From figure 22 it is found that there is a low pressure zone

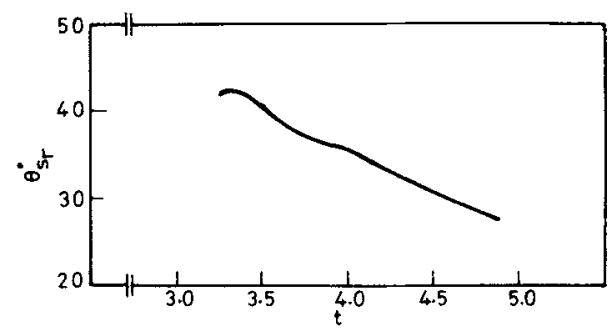

Figure 21. Rear shear layer separation point

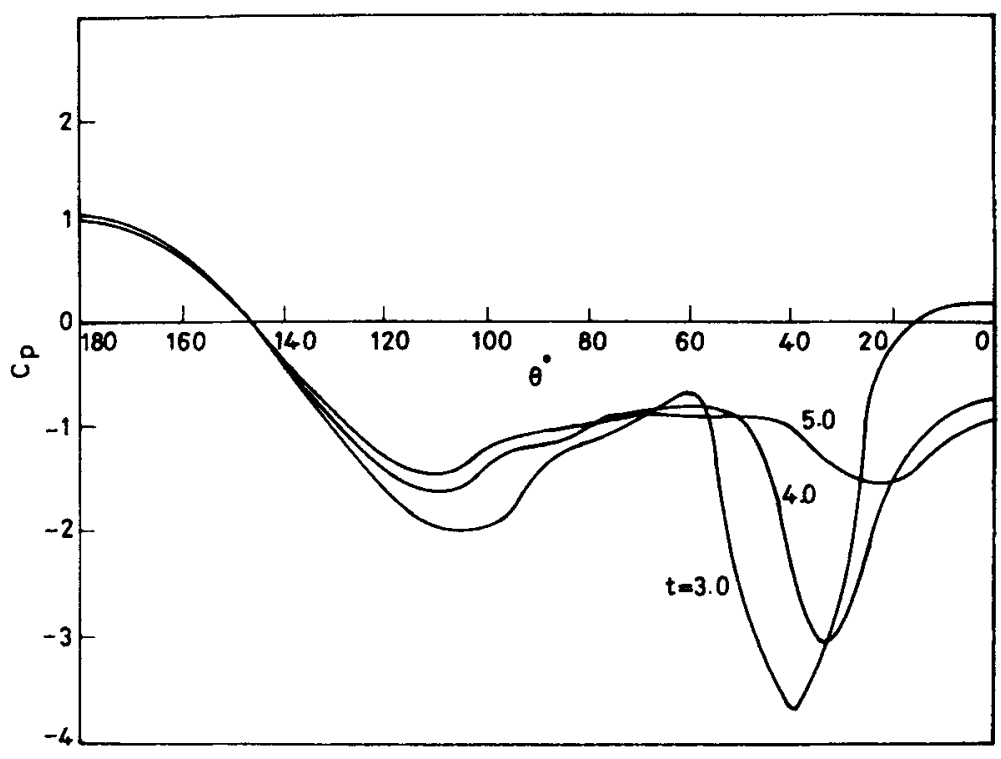

Figure 22. Pressure distribution on cylinder surface 
corresponding to recirculating flow region. The position with minimum pressure is very close to the position with maximum recirculating flow velocity, but they do not coincide. In the early stages of unsteady motion, the negative pressure peak in recirculating flow region may be above the first negative pressure peak near the top of cylinder. With increasing time, the value of first negative pressure peak is decreased, while its position moves forward. From the pressure distributions it is found that from a certain instant after $t=3$, part of the rear shear layer continuously undergoes the action of adverse pressure gradient, so the separation of rear shear layer should be taken into account. The appearance of secondary vortices will obviously change the pressure distributions between two low pressure regions. After a rather long time the tendency of pressure distribution approaches steady state.

Figure 23 shows the potential velocity distributions on the surface of the cylinder at several instants. At $t=1.5$, two stagnation points appear at $\theta=42^{\circ}$ and $\theta=44^{\circ}$. It is shown that a small recirculating flow region begins to appear, after which it expands with time. At $t=3.25$, one end of the recirculating flow region on the cylinder surface reaches the rear stagnation point. After $t=2$ the value of the maximum of recirculating flow velocity may be greater than the value of incoming flow velocity. The maximum velocity on forward surface decreases with time, while its position moves forwards.

Although these pressure and velocity distributions are potential solutions and there are some differences between the present results with real flow field in the region between fore and rear separation points near the surface, it is still helpful in the understanding of the flow near the surface, except in some regions. The positions of fore and rear separation points $\left(S_{f}, S_{r}\right)$ and stagnation points $\left(S_{t}\right)$ on surface are plotted in figure 24. It is also helpful to an understanding of the variation of flow structure near the pair of secondary vortices. Unfortunately, for the pressure and velocity distributions calculated above neither experimental results nor the corresponding exact solutions can be used for comparison. In figure 25 the position of first pressure minimum on the forward surface given in the present work is compared with the results of numerical analysis of the $\mathrm{N}-\mathrm{S}$ equation given by Thoman \& Szewczyk (1969). It is seen that there is a good agreement.

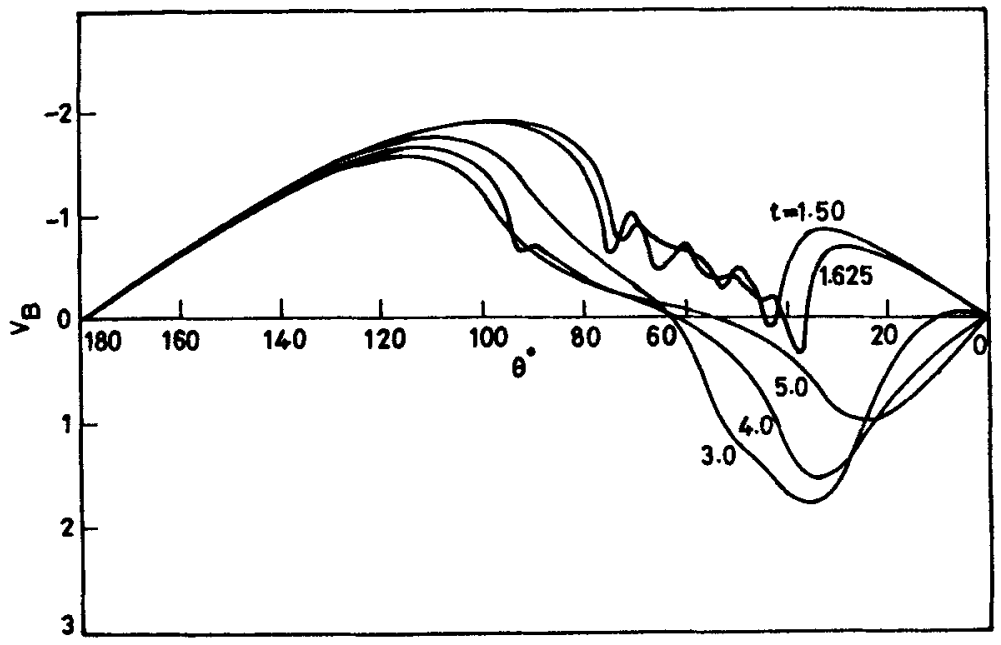

Figure 23. Velocity distributions on cylinder surface 


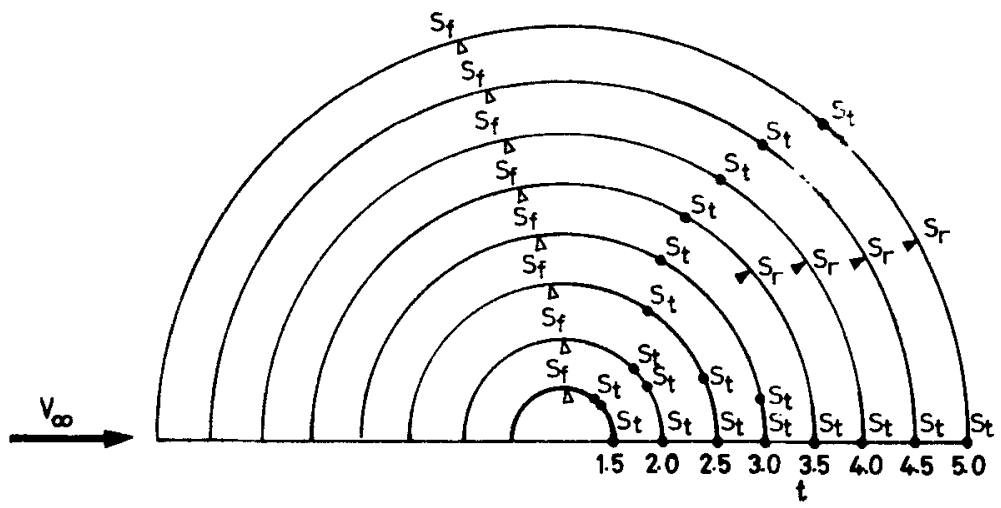

Figure 24. Transient position of separation points $\left(S_{f}\right.$ and $\left.S_{r}\right)$ and stagnation points $\left(S_{t}\right)$ on surface.

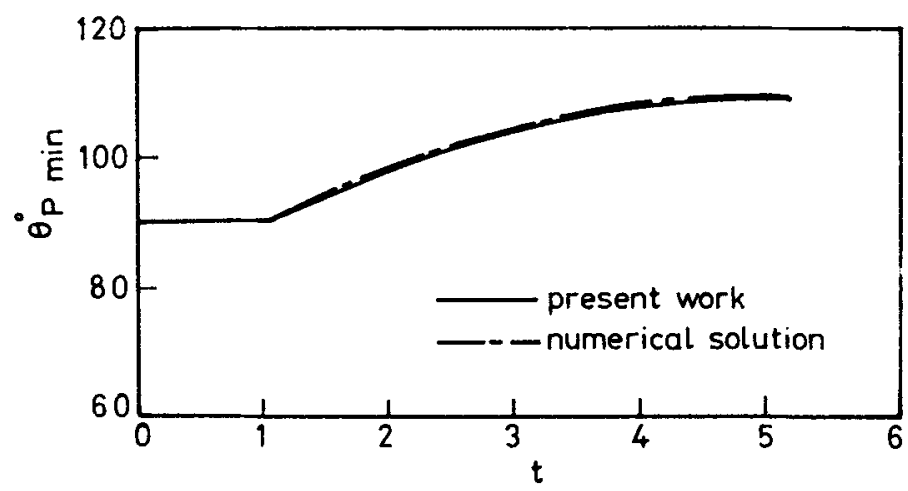

Figure 25. Transient motion of minimum pressure point. The numerical solution is taken from Thoman \& Szewczyk (1969).

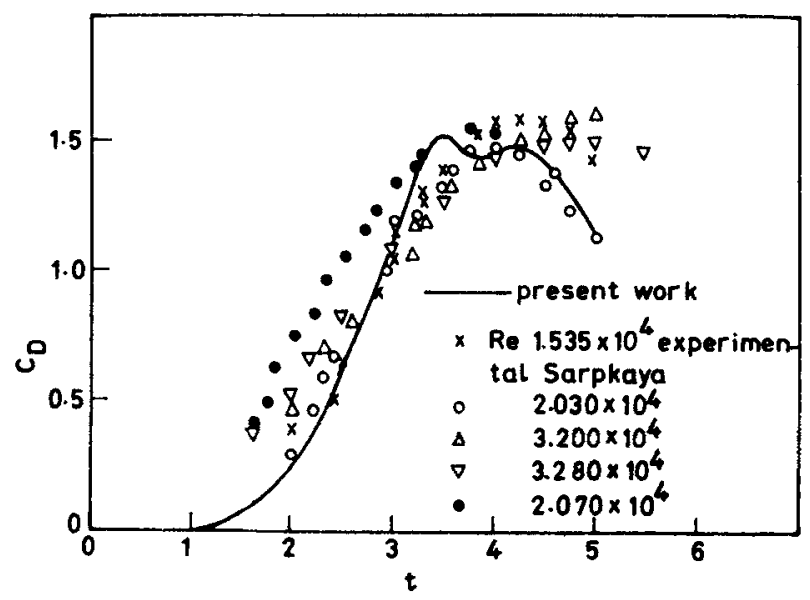

Figure 26. Drag coefficient. 
The variation of form drag coefficient is plotted in figure 26. (At high Reynolds numbers, the part of drag coefficient due to skin friction is of a small quantity as compared with the form drag coefficient, except at the very beginning of unsteady motion.) From Ling \& Yin (1981) we know that if we do not account for the effect of secondary vortices, the drag coefficient would monotonously increase until $C_{D}=2$. Of course, this does not agree with reality. Appearance of secondary vortices will change such a trend of the drag coefficient. The vorticity value in the secondary vortices also affects the drag coefficient. In the present work, the result is in agreement with experiment as shown in figure 26.

\section{Conclusions}

The processes of vortices motion and the development of flow structure in the early stages of unsteady flow around a circular cylinder, especially about the generation and growth of secondary vortices and their effects, have been described in detail. The results of the present work have clearly described the complicated flow development process before the two symmetric main vortices change into asymmetric, i.e. develdpment of symmetric main vortices produces the secondary vortices; due to the effect of growing secondary vortices, each of the symmetric main vortex sheets splits into two parts, a pair of secondary vortices appears. Then the vortex sheets, after breaking off, gradually extend downstream again and the structures of the secondary vortices become relaxed. These results are in good agreement with some flow visualizations. The present authors suggest that when the symmetric main vortices become free vortices, a condition that leads to the instability of the motion of symmetric main vortices should appear. The minimum pressure on cylinder surface and the form drag coefficient calculated by the present work are compared with exact solutions and experimental results and good agreement is obtained. The positions of boundary layer separation are close to those given by some other results. Some flow characteristics on the surface and the development of rear shear layer separation are helpful to the understanding of the variation of the flow field. The present work has provided a mechanism analysis on the flow development in the early processes of formation of Kármán vortex street behind the circular cylinder.

This work was presented at the First Asian Congress of Fluid Mechanics, Bangalore in December 1980. The authors wish to thank Prof. T C Lin for his instructive suggestions and support during the present work. They also thank Prof. C K Wu and Prof. Y K Pien for their kind help.

\section{References}

Bearman P W, Graham J M R 1980 J. Fluid Mech. 99: 225

Chorin A J 1973 J. Fluid Mech. 57: 785

Clements R R 1977 AGARD Lecture Ser. 86

Clements R R, Maull D J 1975 Progr. Aerosp. Sci. 16: 129

Collins W M, Dennis S C R 1973 J. Fluid Mech. 60: 105

Coutanceau M, Bouard R 1980 J. Fluid Mech. 101: 3

Deffenbaugh F D, Marshall F J 1976 AI AA J. 14: 7

Fink P T, Soh W K 1974 Naval hydrodynamics, 10th Symp., Cambridge, Massachusetts 
Gerrard J H 1967 Philos. Trans. R. Soc. London A261: 137

Hiroyuki H, Sadatoshi T 1969 J. Phys. Soc. Jpn 27: 6

Kunio K 1978 J. Phys. Soc. Jpn 45: 1

Lamb H 1945 Hydrodynamics (New York: Dover) 6th ed.

Ling Guo-can, Yin Xie-yuan 1981 Acta Mech. Sin. 2:

Ling Guo-can, Yin Xie-yuan 1982 Acta Mech. Sin. 1:

Moore D W 1974 J. Fluid Mech. 63: 225

Nagata H, Kakehi Y, Tunekawa M, Hasegawa T 1975 Trans. Jpn Soc. Mech. Engrs 41: 341

Patel V A 1976 Comp. Fluids 4: 13

Rosenhead L 1931 Proc. R. Soc. London A134: 170

Saffman P G, Baker G R 1979 Annu. Rev. Fluid Mech. 11: 95

Sarpkaya T 1978 AD-A051781

Sarpkaya T, Shoaff R L 1979 AI AA J. 17: 11

Sears W R, Telionis D P 1975 SIAM J. Appl. Math. 28: 1

Son J S, Hanratty T J 1969 J. Fluid Mech. 35:

Stansby P 1981 The Aeronaut. Q., February

Ta Phuoc Loc 1980 J. Fluid Mech. 100. 9

Taneda S 1972 Recent research on unsteady boundary layer (Quebec Laval University) Vol. 2

Telionis D P, Tsahalis D T 1973 24th Int. Astronautical Congress, Baku, 21 pages

Thoman D C, Szewczyk A A 1969 Phys. Fluids Suppl. 12: II-77-86

White F M 1974 Viscous fluid flow (New York: McGraw Hill)

Yang H T, Bar-Lev 1976 J. Appl. Mech. 43: 1 